\title{
Erratum: Brillouin-Wigner theory for high-frequency expansion in periodically driven systems: Application to Floquet topological insulators [Phys. Rev. B 93, 144307 (2016)]
}

Takahiro Mikami, Sota Kitamura, Kenji Yasuda, Naoto Tsuji, Takashi Oka, and Hideo Aoki

(Received 4 January 2019; published 16 January 2019)

DOI: 10.1103/PhysRevB.99.019902

Just below Eq. (30) of our paper, we assigned "an order 1 for $H(t)$ and the order $k+1$ for $\Lambda^{(k)}(t)$ and $F^{(k)}$." Although this procedure reproduces the correct high-frequency series, the assignment should be corrected to "an order 1 for $H(t)$, the order $k$ for $\Lambda^{(k)}(t)$, and the order $k+1$ for $F^{(k)}$ " in order for the superscript $(k)$ to represent the order in $1 / \omega$. In accordance with this correction, the $k$ sum for $\Lambda(t)$ in Eq. (30) should start from $k=1$ : Namely, Eq. (30) should read

$$
\Lambda(t)=\sum_{k=1}^{\infty} \Lambda^{(k)}(t), \quad F=\sum_{k=0}^{\infty} F^{(k)} .
$$

With this, one can indeed derive Eqs. (32b) and (32c) and (34b)-(34d) correctly. These typographical errors do not alter any conclusions drawn in the paper. 